\title{
On the magnetic mirror effect in Hall thrusters
}

\author{
M. Keidar ${ }^{\text {a) }}$ and I. D. Boyd \\ Department of Aerospace Engineering, University of Michigan, Ann Arbor, Michigan 48109
}

(Received 14 March 2005; accepted 1 August 2005; published online 12 September 2005)

\begin{abstract}
The magnetic mirror effect is studied in the channel of a Hall thruster. It is shown that gradients in magnetic field affect the presheath structure and electric potential distribution. The length of the radial presheath region decreases in the presence of a magnetic field gradient. The two-dimensional potential shape can be affected by proper choice of the magnetic mirror ratio. In particular, it is possible to obtain a concave shape of the potential profile in the channel even in the case of a primarily radial magnetic field. This, in turn, can be used to efficiently control the ion dynamics in the acceleration region. (C) 2005 American Institute of Physics. [DOI: 10.1063/1.2053351]
\end{abstract}

A Hall thruster is a propulsion device in which ions are accelerated in a quasi-neutral plasma. An electric field in the Hall thruster discharge is sustained across the magnetic field. Because of this feature, Hall thrusters offer a much higher thrust density than other types of ion thrusters. Most of the potential drop in this configuration is concentrated in the region of large magnetic fields. This electric field is responsible for ion acceleration. Typically, the radial component of the magnetic field dominates in the acceleration region. Consideration of only the radial component of the magnetic field leads to a one-dimensional picture of the plasma flow in the Hall thruster channel. However, it is an accepted notion that a concave shape of the magnetic field leads to many advantages in thruster operation. ${ }^{1}$ A recent study further explored advantages of using a plasma lens configuration in which the magnetic field has a concave shape in the channel. ${ }^{2}$ A plasma lens magnetic configuration leads to an additional effect of increased magnetic field magnitude near the channel walls in comparison with the channel centerline, the so-called magnetic mirror effect. ${ }^{3}$ However it is not understood how the magnetic mirror effect will affect the potential distribution and ultimately the ion dynamics in the Hall thruster.

The magnetic field distribution is extremely important in Hall thrusters since the electric potential is governed by the magnetic field so that equipotential contours tend to line up with the magnetic field lines. ${ }^{4}$ Indeed, with a correction of a logarithmic factor due to the possible density variation along the magnetic field, this is the case in most conventional Hall thruster channels. ${ }^{5-7}$ In addition, it was recently shown that the electric potential may deviate from the thermalized potential due to radial gradients of magnetic field and electron temperature. ${ }^{8,9}$ Other factors, such as segmented electrodes, can also affect the potential distribution inside the channel. ${ }^{10}$ Understanding the coupling between the potential and magnetic field distributions has tremendous implication for our understanding of Hall thruster operation and may help to formulate any Hall thruster model. Current state-of-the-art Hall thruster models rely on certain assumptions about the potential distribution and, therefore, most predictions are limited to simplified cases. $7,11-14$

In this Letter we attempt to develop a more general formulation for the potential distribution in a Hall thruster taking into account the magnetic mirror effect on the electron

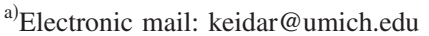

dynamics. Two related effects are considered in this Letter, namely, the effect of the magnetic field gradient on the presheath structure in the 1D radial presheath formulation, and dependence of the 2D potential distribution in the Hall thruster channel on the magnetic field gradient.

We start by formulating a model of the plasma flow between two dielectric walls for a Hall thruster taking into account magnetic field variation in the radial direction as shown schematically in Fig. 1. In this Letter we consider the magnetic mirror effect by taking into account only the radial component of the magnetic field.

Increase of the magnetic field magnitude from the channel centerline towards the wall leads to an additional force that acts on the electrons. Let us consider a weakly divergent magnetic field, i.e., radial magnetic field variation along $r$ independent of $z$ as shown schematically in Fig. 1. In this case the adiabatic invariant is conserved along the magnetic field that leads to the so-called magnetic mirror effect. Under these conditions it is simple to show that the additional force acting on the electrons depends on the electron energy. ${ }^{15}$ In this case the electron momentum equation along the magnetic field in the absence of current along the magnetic field can be written as follows:

$$
0=\operatorname{en} \nabla \varphi-n k T_{e} \frac{1}{B} \nabla B-\nabla\left(n k T_{e}\right),
$$

where $n$ is the electron density, $\varphi$ is the potential, $B$ is the magnetic field, and $T_{e}$ is the electron temperature. In the case of small density gradient, the potential drop is such that potential is more positive in the high magnetic field region ${ }^{16}$

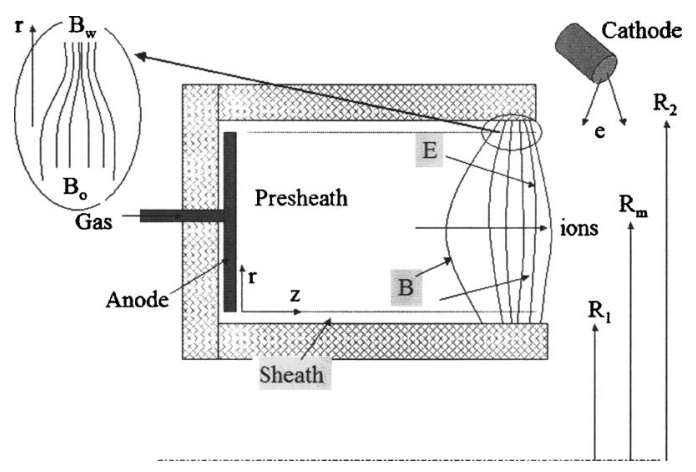

FIG. 1. Schematic of the Hall thruster. In the enlarged area the simplified magnetic field geometry is shown. 


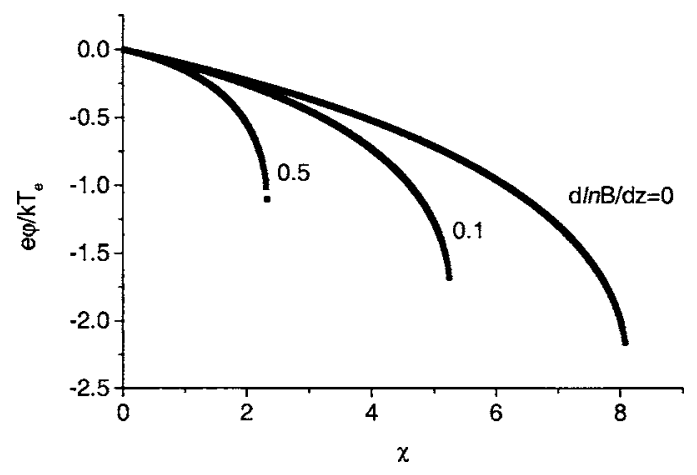

FIG. 2. Potential distribution in the presheath. $\alpha=1$.

$$
\varphi_{\max }-\varphi_{\min }=\frac{k T_{e}}{e} \ln \frac{B_{\max }}{B_{\min }} .
$$

Based on this effect a so-called End-Hall ion source was developed. ${ }^{16}$ In this Letter, we study the influence of the magnetic mirror effect on the plasma flow and plasma-wall transition in Hall thrusters.

Let us start from analysis of the quasi-neutral plasma (presheath) problem in the Hall thruster. It was shown previously that the presheath scale length becomes comparable to the channel width under typical conditions of the Hall thruster plasma flow. ${ }^{7}$ Thus, a model for the quasi-neutral plasma region is extended up to the sheath edge in order to provide boundary conditions at the plasma-sheath interface as shown in Fig. 1. In this model we will consider a 1D presheath structure in the radial direction between the plasma bulk and the dielectric wall.

The presheath model is based on the assumption that the quasi-neutral region length (i.e., the channel width, Fig. 1) is much larger than the Debye radius and, therefore, we will assume $Z_{i} n_{i}=n_{e}=n$, where $Z_{\mathrm{i}}$ is the ion mean charge, $n_{i}$ is the ion density, and $n_{e}$ is the electron density. For simplicity only single charge ions are considered in this paper $\left(Z_{i}=1\right)$. Electrons are assumed to be collisionless. A condition that the magnetic field has only a radial component, $B_{r}=B$, is imposed. In the hydrodynamic model it is additionally assumed: (i) the system reaches a steady state; (ii) the electron component is not inertial, i.e., $\left(V_{e} \nabla\right) \cdot V_{e}=0$; (iii) ionization is neglected. Under these assumptions, the following system of equations describes the ion component of the quasi-neutral plasma:

$$
n m_{i}\left(V_{i} \nabla\right) V_{i}=e n E-\nabla P_{i}-n m_{i} V_{i} v_{c}
$$

$$
\nabla\left(n V_{i}\right)=0
$$

where $P_{i}$ is the ion pressure and $v_{c}$ is the total ion collision frequency. Typically, in Hall thrusters the ion temperature is much smaller than the electron temperature and, therefore, ion pressure can be neglected. By combining Eqs. (1), (3), and (4) one can obtain the equation for the ion velocity in the presheath,

$$
\left(V-\frac{C_{s}^{2}}{V}\right) \frac{d V}{d r}=-v_{c} V-\frac{k T_{e}}{m_{i}} \frac{1}{B} \frac{d B}{d r},
$$

where $C_{s}$ is the Bohm velocity, $C_{s}=\sqrt{k T_{e} / m_{i}}$. Along the magnetic field the equation for the potential distribution reads
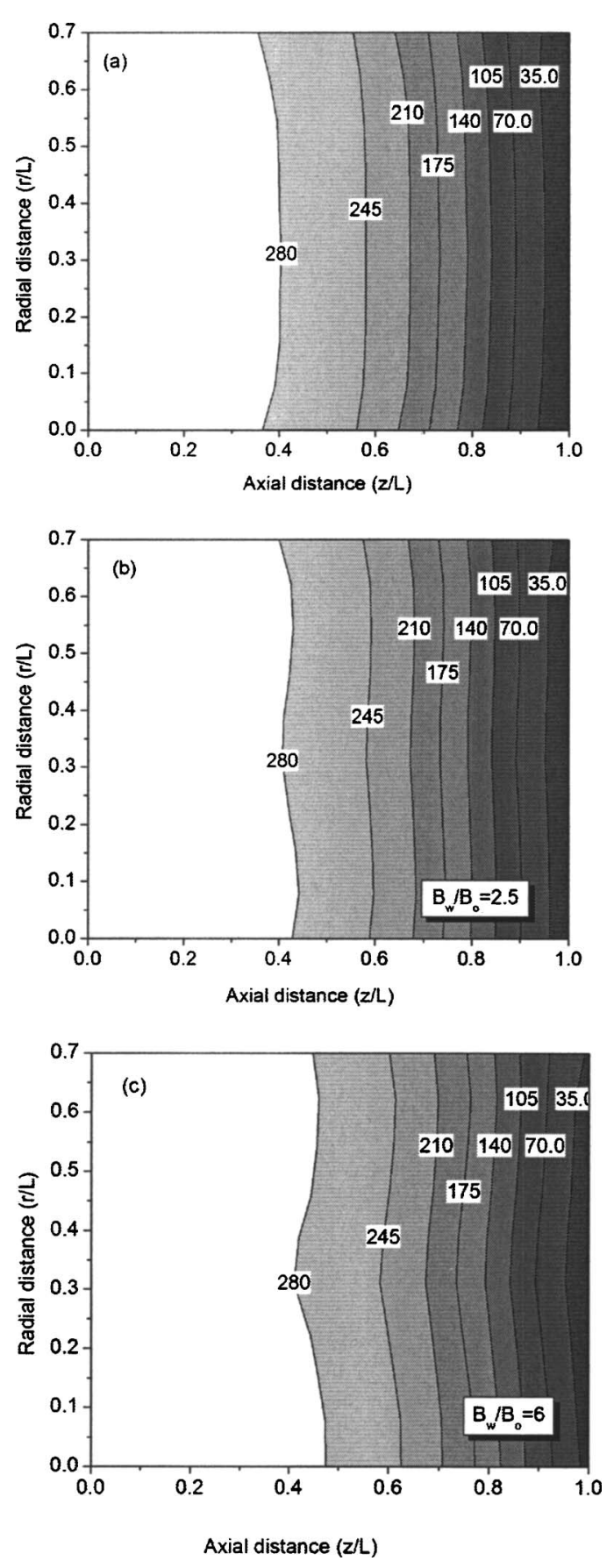

FIG. 3. 2D potential profile in the SPT-100 type Hall thruster channel simulated based on hydrodynamic model. $B_{w} / B_{o}$ is the ratio of the magnetic field near the wall to the magnetic field in the middle of the channel. $L$ is the length of the Hall thruster channel. Coordinate origin ( 0 is the anode plane) is upstream at the inner wall of the channel as shown in Fig. 1.

$$
0=\frac{d \varphi}{d r}-\frac{k T_{e}}{e B} \frac{d B}{d r}-\frac{k T_{e}}{e n} \frac{d n}{d r}
$$

We use the following normalized variables: $u=V / C_{s}, \phi$ $=e \varphi / k T_{e}, \alpha=v_{c} \rho_{e} / C_{s}, \rho_{e}=m_{e} C_{s} /(e B), \chi=r / \rho_{e}$. After normalization the system of equation will have the following form:

$$
\frac{1-u^{2}}{u} \frac{d u}{d \chi}=\alpha u+\frac{d \ln B}{d \chi},
$$




$$
\frac{d \phi}{d \chi}=\frac{d \ln B}{d \chi}+\frac{d \ln n}{d \chi} .
$$

In the solution of the system of Eqs. (7) and (8), the plasma cannot overcome the ion sound speed as follows from Eq. (7). Therefore, the system of Eqs. (7) and (8) determines the plasma parameter distribution in the presheath up until the presheath-sheath interface.

The potential distribution in the presheath is shown in Fig. 2 with magnetic field gradient as a parameter. One can see that the potential drop in the presheath decreases with increased magnetic field gradient. For instance when $(1 / B)$ $\times(d B / d \chi)$ increases from 0 to 0.5 potential drop decreases by factor of 2 . In addition, the length of the presheath decreases with increase of the magnetic field gradient as shown in Fig. 2.

It is important to note that the potential distribution along the magnetic field can be determined from Eq. (6). This is the generalized form of the so-called "thermalized" potential introduced by Morozov. ${ }^{4,5}$ It should be pointed out that all state-of-the-art Hall thruster models assume the "thermalized" potential along the magnetic field line. ${ }^{7,11-14}$ However, it is important to understand how the magnetic field gradient affects the potential distribution along the magnetic field, since it directly affects the ion dynamics in the Hall thruster channel.

In order to study this effect we use a two-dimensional model of the plasma flow in a Hall thruster described elsewhere ${ }^{7}$ and having the following features. A hydrodynamic model is employed in a 2-D domain assuming that the system reaches a steady state. In order to simplify the problem without missing the major physical effects, we consider one-dimensional flow of the neutrals. The momentum and mass conservation equations for ions and neutrals and energy balance are considered. Electron conductivity is specified to be governed by Bohm type anomalous conductivity across the magnetic field. ${ }^{7,13,14}$ We modified this model by taking into account the magnetic field gradient along the magnetic field. In this case the potential distribution is calculated according to Eq. (1).

Calculated 2D potential distribution in the Hall thruster channel is shown in Fig. 3 with magnetic mirror ratio (ratio of the magnetic field near the wall to the magnetic field in the center of channel, $B_{w} / B_{o}$ ) as a parameter. One can see that the magnetic field gradient significantly affects the potential distribution. Higher magnetic field gradient leads to more positive potential in the high magnetic field region. In turn this changes the shape of the equipotential lines. Usually, the magnetic mirror ratio is about 2-3 (Refs. 2 and 3). However taking into account the concave shape of the magnetic field (i.e., extending our consideration beyond the simplified case with only radial magnetic field component) the magnetic mirror ratio can be up to 6 along a magnetic field line. ${ }^{2,3}$ It should be noted that a high magnetic mirror ratio leads to a concave potential profile even in the case of a mainly radial magnetic field component.

In conclusion, in this Letter we considered an effect of the magnetic mirror on several aspects of the plasma flow in a Hall thruster channel. The magnetic field gradient affects several important flow characteristics, such as the radial presheath potential drop, the presheath length, and the twodimensional potential shape in the channel. In particular, it was shown that a concave shape of the potential can be obtained even in the case of a magnetic field having primarily radial component. This effect is important for ion dynamics since ions follow the electric field in the absence of collisions as happens typically in the acceleration region. Consideration of the magnetic field gradient in the model formulation represents an important departure from the current stateof-the-art modeling level for Hall thrusters.

The authors thank Professor Alec Gallimore, Dr. Richard Hofer, and Jesse Linnell for very fruitful discussion. This work was supported by AFOSR Grant No. FA9550-05-10042.

${ }^{1}$ V. Kim, J. Propul. Power 14, 736 (1998).

${ }^{2}$ R. Hofer, P. Peterson, A. D. Gallimore, and R. S. Jankovsky, 27th International Electric Propulsion Conference, Pasadena, CA, 15-19 October 2001 (Electric Rocket Propulsion Society, Cleveland, OH 2003), IEPC Paper 2001-036.

${ }^{3}$ R. Hofer and A. D. Gallimore, Proceedings of 38th Joint Propulsion Conference and Exhibit, July 2002, Indianapolis, IN (American Institute of Aeronautics and Astronautics, Reston, VA, 2002), AIAA Paper-20024111.

${ }^{4}$ A. I. Morozov, Sov. Phys. Dokl. 10, 775 (1966).

${ }^{5}$ A. I. Morozov and V. V. Savelyev, in Review of Plasma Physics, edited by B. B. Kadomtsev and V. D. Shafranov (Consultant Bureau, New York, 2000), Vol. 21, p. 203.

${ }^{6}$ V. V. Zhurin, H. R. Kaufman, and R. S. Robinson, Plasma Sources Sci. Technol. 8, 1 (1999).

${ }^{7}$ M. Keidar, I. D. Boyd, and I. I. Beilis, Phys. Plasmas, 91675 (2001)

${ }^{8}$ M. Keidar, A. D. Gallimore, Y. Raitses, and I. D. Boyd, Appl. Phys. Lett. 85, 2481 (2004).

${ }^{9}$ Y. Raitses, M. Keidar, D. Staack, and N. J. Fisch, J. Appl. Phys. 92, 4906 (2002).

${ }^{10}$ Y. Raitses, D. Staack, L. Dorf, and N. J. Fisch, Proceeding of 39th Joint Propulsion Conference, 20-23 July 2003, Huntsville, AL (American Institute of Aeronautics and Astronautics, Reston, VA 2002), AIAA paper 2003-5153

${ }^{11}$ G. J. M. Hagelaar, J. Bereilles, L. Garrigues, and J.-P. Boeuf, J. Appl. Phys. 93, 67 (2003).

${ }^{12}$ L. Garrigues, G. J. M. Hagelaar, J. Bareilles, C. Boniface, and J. P. Boeuf, Phys. Plasmas 10, 4886 (2003).

${ }^{13}$ J. W. Koo, and I. D. Boyd, Comput. Phys. Commun. 164, 442 (2004).

${ }^{14}$ J. M. Fife, Hybrid-PIC Modeling and Electrostatic Probe Survey of Hall Thrusters, Ph.D. thesis (MIT, 1998).

${ }^{15}$ H. Muta, N. Itagaki, and Y. Kawai, Vacuum 66, 209 (2002).

${ }^{16}$ H. Kaufman, R. S. Robinson, and R. I. Seddon, J. Vac. Sci. Technol. A 5(4), 2081 (1987). 